DSF-20/03

LAPTH-987/03

cond-mat/0306636

\title{
Sum rules for free energy and frequency distribution of DNA dinucleotides
}

\author{
L. Frappat ${ }^{a c}$, A. Sciarrino ${ }^{b}$ \\ ${ }^{a}$ Laboratoire d'Annecy-le-Vieux de Physique Théorique LAPTH, \\ CNRS, UMR 5108, and Université de Savoie, \\ BP 110, F-74941 Annecy-le-Vieux Cedex, France \\ ${ }^{b}$ Dipartimento di Scienze Fisiche, Università di Napoli "Federico II" \\ and I.N.F.N., Sezione di Napoli, \\ Complesso Universitario di Monte S. Angelo, \\ Via Cintia, I-80126 Naples, Italy \\ ${ }^{c}$ Member of Institut Universitaire de France
}

\begin{abstract}
The large discrepancy between the values of the free energy for DNA dinucleotides (or dimers) measured by different teams has raised a yet unsettled debate. Here the free energy is fitted by a three parameter empiric formula derived in the framework of the crystal basis model of genetic code. Sum rules are derived and compared satisfactorily with the data. On the basis of theoretical and phenomenological arguments, a relation between the correlation functions of dimer distribution and the free energy is assumed. From consistency conditions, sum rules are derived. A check of these conditions with different samples of experimental data is performed, allowing us to argue on the reliability of the different sets of experimental data.
\end{abstract}

PACS number: 87.10.+e, 02.10.-v 


\section{Introduction}

The importance of the computation of the free energy $\Delta G^{0}$ and enthalpy $\Delta H^{0}$ for DNA dinucleotides or dimers was recognized in the eighties by many authors and several experimental measures have been performed. The experimental values however range in an unacceptable wide range. A few years ago SantaLucia [1] has performed an accurate analysis and comparison of the data from seven laboratories (see Table 6 taken from ref. [1], where we have replaced the original values of the column Benight [14] with the more recent ones [13]), reaching the conclusion that six of the studies were actually in agreement and providing explanations for the discrepancies. In an attempt to settle by thermodynamics arguments the controversy, Miramontes and Cocho [2] have analysed quite recently the same set of data by assuming a relation between the correlation function of the dimers and their free energy, reaching the conclusion that the most reliable set of values is just the one which was excluded by SantaLucia. Indeed in ref. [2] a linear relation between the correlation function for the dimer and the corresponding free energy was postulated, which allowed these authors to determine which set of experimental data was in better agreement with the postulated relation. A shortcoming of this analysis is that the sum of the free energies for strong dimers does not satisfy an identity derived from the postulated equation. The purpose of this work is to come back to this controversial question. First, we propose a theoretical formula to compute the free energy, from which sum rules are derived and compared with the values of experimental data. Second, we motivate the assumption of a relation between the correlation function and the free energy, different from the one assumed in [2], which satisfies trivial identities required by the definition of the correlation functions. We make several consistency checks and we try to determine the reliability of the experimental values, comparing with the calculated values of the correlation matrix in [2].

\section{$2 \quad$ Fit for the free energy}

Let us recall that a mathematical framework was proposed [3], in which the codons appear as composite states of nucleotides. The four nucleotides being assigned to the fundamental irreducible representation of the quantum group $\mathcal{U}_{q}\left(s l_{H}(2) \oplus s l_{V}(2)\right)$ in the limit $q \rightarrow 0$ (the indices $H$ and $V$ distinguish the two $\left.s l(2)\right)$, a sequence of $N$ nucleotides is described by a pure state in the $N$-fold tensor product of the fundamental representation. In particular, dimers or dinucleotides are obtained as the two-fold tensor product, the labels specifying the irreducible representation to which they belong being given in Table 5. In ref. [3] we have fitted old experimental data of the free energy $\Delta G_{37}^{0}$ (for simplicity we will omit the temperature label in the following) for RNA dinucleotides with a 4 parameter formula built up with the generators of $\mathcal{U}_{q \rightarrow 0}\left(s l_{H}(2) \oplus s l_{V}(2)\right)$ and in [4] the more recent data of [5] have been fitted with the following 2 parameter formula

$$
\Delta G^{0}=\alpha+\beta\left(C_{H}+C_{V}\right) J_{3 H}
$$

where $J_{3 X}(X=H$ or $V)$ stands for the diagonalized $\operatorname{sl}(2)_{X}$ generator and $C_{X}$ is the Casimir operator of $\mathcal{U}_{q \rightarrow 0}\left(\operatorname{sl}(2)_{X}\right)$ for the considered dimer $i j$. Let us recall that the Casimir operator eigenvalue in the $J$-representation is $J(J+1)$. In order not to overload the notation, here and in the following, we will not explicitly write the labels of the dimer, if not necessary to identify a specific dimer.

Here we propose for the DNA dinucleotides a 3 parameter formula, which is a generalisation of eq. (11):

$$
\Delta G^{0}=\alpha_{0}+\alpha_{1} J_{3 H}+\alpha_{2}\left(J_{3 V}\right)^{2}\left(2 J_{3 H}+1\right)
$$


This equation leads to the following theoretical values of the dimer free energies $\Delta G^{0}$ in terms of the parameters $\alpha_{0}, \alpha_{1}, \alpha_{2}$ :

\begin{tabular}{|lc|cc|}
\hline $\mathrm{AA} / \mathrm{TT}$ & $\alpha_{0}-\alpha_{1}-\alpha_{2}$ & $\mathrm{CT} / \mathrm{GA}$ & $\alpha_{0}+\alpha_{2}$ \\
$\mathrm{AT} / \mathrm{TA}$ & $\alpha_{0}-\alpha_{1}$ & $\mathrm{GA} / \mathrm{CT}$ & $\alpha_{0}+\alpha_{2}$ \\
$\mathrm{TA} / \mathrm{AT}$ & $\alpha_{0}-\alpha_{1}$ & $\mathrm{GG} / \mathrm{GC}$ & $\alpha_{0}+\alpha_{1}$ \\
$\mathrm{CA} / \mathrm{GT}$ & $\alpha_{0}$ & GC $/ \mathrm{CG}$ & $\alpha_{0}+\alpha_{1}$ \\
$\mathrm{GT} / \mathrm{CA}$ & $\alpha_{0}$ & $\mathrm{GG} / \mathrm{CC}$ & $\alpha_{0}+\alpha_{1}+3 \alpha_{2}$ \\
\hline
\end{tabular}

A best-fit procedure allows one to evaluate these parameters as follows:

$$
\alpha_{0}=\frac{1}{116}\left(14 N_{1}+4 N_{2}-6 N_{3}\right), \quad \alpha_{1}=\frac{1}{116}\left(4 N_{1}+26 N_{2}-10 N_{3}\right), \quad \alpha_{2}=\frac{1}{116}\left(-6 N_{1}-10 N_{2}+15 N_{3}\right)
$$

where (we specify by a couple of indices the free energy of a dinucleotide)

$$
\begin{aligned}
& N_{1}=\Delta G_{G G}^{0}+\Delta G_{C G}^{0}+\Delta G_{G C}^{0}+\Delta G_{C T}^{0}+\Delta G_{G A}^{0}+\Delta G_{G T}^{0}+\Delta G_{C A}^{0}+\Delta G_{T A}^{0}+\Delta G_{A T}^{0}+\Delta G_{A A}^{0} \\
& N_{2}=\Delta G_{G G}^{0}+\Delta G_{G C}^{0}+\Delta G_{C G}^{0}-\Delta G_{A A}^{0}-\Delta G_{A T}^{0}-\Delta G_{T A}^{0} \\
& N_{3}=3 \Delta G_{G G}^{0}+\Delta G_{C T}^{0}+\Delta G_{G A}^{0}-\Delta G_{A A}^{0}
\end{aligned}
$$

\begin{tabular}{|c|c|c|c|c|c|c|c|c|}
\hline & $\begin{array}{c}\text { Gotoh } \\
6]\end{array}$ & $\begin{array}{c}\text { Vologodskii } \\
{[7}\end{array}$ & $\begin{array}{c}\text { Breslauer } \\
8\end{array}$ & $\begin{array}{c}\text { Delcourt } \\
9]\end{array}$ & $\begin{array}{c}\text { SantaLucia } \\
\text { [10 }\end{array}$ & $\begin{array}{c}\text { Sugimoto } \\
{[11}\end{array}$ & $\begin{array}{c}\text { Unified } \\
12\end{array}$ & $\begin{array}{c}\text { Benight } \\
13\end{array}$ \\
\hline$\alpha_{0}$ & 0.98 & 1.37 & 1.89 & 1.24 & 1.53 & 1.71 & 1.47 & 1.35 \\
\hline$\alpha_{1}$ & 0.70 & 0.60 & 0.99 & 0.61 & 0.66 & 0.81 & 0.73 & 0.54 \\
\hline$-\alpha_{2}$ & 0.14 & 0.12 & 0.18 & 0.09 & 0.15 & 0.16 & 0.14 & 0.03 \\
\hline$s^{2}$ & 0.0015 & 0.0011 & 0.1577 & 0.0014 & 0.0114 & 0.0199 & 0.0070 & 0.0069 \\
\hline$\chi^{2}$ & 0.0243 & 0.0099 & 1.0001 & 0.0167 & 0.0753 & 0.0992 & 0.0821 & 0.0590 \\
\hline
\end{tabular}

Hence we get for the different studies, see Table [6, the best-fit values of the parameters $\alpha_{0}, \alpha_{1}, \alpha_{2}$ :

The last two rows correspond to the square mean deviation $s^{2}=\frac{1}{N} \sum\left(y_{\text {exp }}-y_{t h}\right)^{2}(N$ is the number of points, here 10) and to $\chi^{2}=\sum\left(y_{\text {exp }}-y_{t h}\right)^{2} / y_{t h}$. Evaluation of the incomplete Gamma function, which is an estimate of the goodness-of-fit, shows that the fit is good with a confidence level greater than $95 \%$. Table 7 gives the fitted absolute values for dimer free energy parameters $\Delta G^{0}$ corresponding to the different samples. From an inspection of the values of $s^{2}$ and $\chi^{2}$, one sees that eq. (2) is well fitted by the different sets of experimental data, except by the ones from Breslauer.

\section{Sum rules}

We derive from eq. (2) a set of identities and sum rules. First, it is clear that

$$
\Delta G_{i j}^{0}=\Delta G_{j i}^{0} \quad \text { and } \quad \sum_{j=A, C, G, T} \Delta G_{i j}^{0}=\sum_{j=A, C, G, T} \Delta G_{j i}^{0}
$$

In particular we get

$$
\begin{aligned}
\sum_{j=A, C, G, T} \Delta G_{C j}^{0} & =\sum_{j=A, C, G, T} \Delta G_{G j}^{0}=4 \alpha_{0}+2 \alpha_{1}+4 \alpha_{2} \\
\sum_{j=A, C, G, T} \Delta G_{A j}^{0} & =\sum_{j=A, C, G, T} \Delta G_{T j}^{0}=4 \alpha_{0}-2 \alpha_{1} \\
\sum_{i, j=A, C, G, T} \Delta G_{i j}^{0} & =16 \alpha_{0}+8 \alpha_{2}
\end{aligned}
$$


In Table 1 we report the experimental values computed using the values of Table 6, Note that in ref. 2 the existence of the sum rules eqs. (6) and (7) was already remarked, but the two equations should have the same values, which is actually not the case.

Table 1: Experimental values of the sums of free energies [see eq. (15)].

\begin{tabular}{|c|cccccccc|}
\hline & Gotoh & Vologodskii & Breslauer & Delcourt & SantaLucia & Sugimoto & Unified & Benight \\
\hline$\sum_{i} \Delta G_{C i}^{0}$ & 4.72 & 6.16 & 9.18 & 5.78 & 6.72 & 8.10 & 6.74 & 6.54 \\
$\sum_{i} \Delta G_{G i}^{0}$ & 4.77 & 6.20 & 8.11 & 5.80 & 6.94 & 7.40 & 6.82 & 6.26 \\
$\sum_{i} \Delta G_{T i}^{0}$ & 2.55 & 4.27 & 5.63 & 3.68 & 5.08 & 5.30 & 4.33 & 4.45 \\
$\sum_{i} \Delta G_{A i}^{0}$ & 2.51 & 4.21 & 5.33 & 3.74 & 4.51 & 5.10 & 4.60 & 4.27 \\
\hline
\end{tabular}

Due to the complementarity rule, one has

$$
\begin{aligned}
\sum_{i=A, C, G, T} \Delta G_{C i}^{0} & =\sum_{i=A, C, G, T} \Delta G_{i G}^{0} & \text { and } & \sum_{i=A, C, G, T} \Delta G_{G i}^{0} & =\sum_{i=A, C, G, T} \Delta G_{i C}^{0} \\
\sum_{i=A, C, G, T} \Delta G_{A i}^{0} & =\sum_{i=A, C, G, T} \Delta G_{i U}^{0} & \text { and } & \sum_{i=A, C, G, T} \Delta G_{U i}^{0} & =\sum_{i=A, C, G, T} \Delta G_{i A}^{0}
\end{aligned}
$$

Now we derive also news sum rules

$$
\begin{aligned}
& \Delta G_{C G}^{0}+\Delta G_{T A}^{0}=2 \Delta G_{T G}^{0}=2 \Delta G_{A C}^{0} \\
& \Delta G_{C C}^{0}+\Delta G_{T T}^{0}=2 \Delta G_{T C}^{0}=2 \Delta G_{G A}^{0} \\
& \Delta G_{C C}^{0}+\Delta G_{A A}^{0}=2 \Delta G_{T C}^{0}=2 \Delta G_{A G}^{0}
\end{aligned}
$$

We report in Table 2 a comparison with the experimental data, making an average of the different experimental values, theoretically equal due to eq. (2), i.e.

$$
\begin{aligned}
& S_{1}=\Delta G_{C G}^{0}+\Delta G_{T A}^{0}+\Delta G_{G C}^{0}+\Delta G_{A T}^{0}-\Delta G_{T G}^{0}-\Delta G_{G T}^{0}-\Delta G_{A C}^{0}-\Delta G_{C A}^{0}=0 \\
& S_{2}=\Delta G_{C C}^{0}+\Delta G_{T T}^{0}+\Delta G_{G G}^{0}+\Delta G_{A A}^{0}-\Delta G_{C T}^{0}-\Delta G_{T C}^{0}-\Delta G_{A G}^{0}-\Delta G_{G A}^{0}=0
\end{aligned}
$$

Table 2: Sum rules for free energies [see eqs. (14)-(15)].

\begin{tabular}{|c|cccccccc|}
\hline & Gotoh & Vologodskii & Breslauer & Delcourt & SantaLucia & Sugimoto & Unified & Benight \\
\hline$S_{1}$ & -0.07 & 0.08 & 2.19 & 0.10 & -0.09 & 0.50 & 0.09 & -0.32 \\
$S_{2}$ & -0.22 & 0.24 & 3.30 & -0.14 & 0.34 & 0.60 & 0.52 & 0.36 \\
\hline
\end{tabular}

As it can be seen the sum rules are reasonably well satisfied, except for the data of Breslauer. However we cannot make any statement on the reliability of the different experimental data on the basis of the accuracy by which they fit our empirical formula eq. (2).

\section{Dinucleotide distribution}

In order to settle on more theoretical ground our analysis, we consider the dimer correlation function. In 2] the dimer distribution was characterized by the correlation function

$$
\Gamma_{i j}=f_{i j}-f_{i} f_{j}
$$


where the labels $i, j$ denote the nucleotides, $i, j \in\{A, C, G, T\}$, and $f_{i}\left(f_{i j}\right)$ denote the frequency of the $i$ nucleotide ( $i j$ dinucleotide). From eq. (16), it follows

$$
\sum_{i=A, C, G, T} \Gamma_{i j}=\sum_{j=A, C, G, T} \Gamma_{i j}=0
$$

In 2] the following relation between $\Gamma_{i j}$ and the free energy $\Delta G^{0}$ was assumed:

$$
\Gamma_{i j}=a+b \Delta G_{i j}^{0}
$$

where $a$ and $b$ are biological species dependent parameters. Inserting eq. (2) into eq. (17) one gets the identity

$$
4 a+b \sum_{j=A, C, G, T} \Delta G_{i j}^{0}=0 \Rightarrow \sum_{j=A, C, G, T} \Delta G_{i j}^{0}=\text { const. for all } i
$$

In ref. [2], from the data reported in Table 6, except the last column which was not considered, the authors show that eq. (19) was satisfied by the weak dimers only, i.e. with label $i \in\{A, T\}$. Let us remark: i) that the statistical mechanics motivation which led the authors to postulate eq. (2) holds for an isolated system, which is not the case for a dimer inserted in a DNA strand; ii) the computed values of the correlation matrix, see Table 3 of [2], for the same biological species, show, in many cases, a much larger variation than the corresponding variation of the free energy, changing the $i j$ dimer; iii) our empirical formula eq. (2) predicts the dimers $i j$ and $j i$ to have the same free energy, which is approximately true (see Table 6 ), while on the contrary the correlation function $\Gamma_{i j}$ is generally non symmetric. From the above remarks we assume the following relation between $\Gamma_{i j}$ and $\Delta G_{i j}^{0}$ :

$$
\Gamma_{i j}=a+b\left(\Delta G_{i j}^{0}-\frac{1}{4} \sum_{k=A, C, G, T}\left(\Delta G_{k i}^{0}+\Delta G_{j k}^{0}\right)\right)+\left(1-\delta_{i j}\right) h_{i j}
$$

where $h_{i j}$ are biological species dependent real coefficients. The complementarity implies that the coefficients $h_{i j}$ and $h_{\bar{\jmath}}$ are equal for two complementary dimers $i j$ (from $5^{\prime}$ to $3^{\prime}$ ) and $\bar{\imath}$ (from $3^{\prime}$ to $5^{\prime}$ ), so there is only 8 coefficients $h_{i j}$.

The corrective term in the free energy can be considered as a "penalty" due to the interaction of the nucleotides of the dimer with the two nearest neighbour nucleotides in the strand, assumed uniformly distributed.

Since the correlation coefficient $\Gamma_{i j}$ has to satisfy the sum rule (17) by definition, one is led to the constraints $(\forall j)$

$$
\begin{aligned}
0 & =4 a+b \sum_{i=A, C, G, T}\left(\Delta G_{i j}^{0}-\Delta G_{j i}^{0}\right)-\frac{b}{4} \sum_{k, i=A, C, G, T} \Delta G_{k i}^{0}+\sum_{i=A, C, G, T}\left(1-\delta_{i j}\right) h_{i j} \\
& =4 a+b \sum_{i=A, C, G, T}\left(\Delta G_{j i}^{0}-\Delta G_{i j}^{0}\right)-\frac{b}{4} \sum_{k, i=A, C, G, T} \Delta G_{i k}^{0}+\sum_{i=A, C, G, T}\left(1-\delta_{i j}\right) h_{i j}
\end{aligned}
$$

Eqs. (51)-(8) imply for any pair $(i, j)$ of nucleotides

$$
2 b\left(2 \alpha_{0}+\alpha_{2}\right)-4 a=\sum_{k=A, C, G, T}\left(1-\delta_{i k}\right) h_{i k}=\sum_{k=A, C, G, T}\left(1-\delta_{k j}\right) h_{k j}
$$


As eq. (22) gives 4 independent relations, we are left with 4 parameters $h_{i j}$. We remark that in eq. (20) only the following combinations of $a, b$ and $\alpha_{i}$ parameters appear in the free energy term:

$$
x=a-b \alpha_{0} \quad \text { and } \quad y=b \alpha_{2}
$$

We then deduce from the 4 constraints (22) the following relations among the coefficients $h_{i j}$ (we choose $\left.h_{C A}, h_{C T}, h_{C G}, h_{A C}, h_{T C}, h_{G C}, h_{A T}, h_{A T}\right)$

$$
\begin{aligned}
& h_{C G}+h_{G C}-h_{A T}-h_{T A}=0 \\
& h_{T C}-h_{C T}+h_{G C}-h_{A T}=0 \\
& h_{C A}-h_{A C}+h_{C T}-h_{T C}+h_{C G}-h_{G C}=0
\end{aligned}
$$

Using eq. (20) we can replace the following equations by sum rules for the corresponding correlation coefficients:

$$
\begin{aligned}
& \Gamma_{C G}+\Gamma_{G C}-\Gamma_{A T}-\Gamma_{T A}=-4 y=2\left(\Gamma_{A A}-\Gamma_{C C}\right) \\
& \Gamma_{C T}-\Gamma_{T C}+\Gamma_{C G}-\Gamma_{T A}=-2 y=\Gamma_{A A}-\Gamma_{C C} \\
& \Gamma_{C A}-\Gamma_{A C}+\Gamma_{C T}-\Gamma_{T C}+\Gamma_{C G}-\Gamma_{G C}=0
\end{aligned}
$$

The above equations are well satisfied (within $<5 \%$ ) by the experimental data, see Table 3 of [2], therefore we conclude that our parametrization (20) for the correlation function is satisfactory and we can carry on our analysis.

Consider the following differences of the correlation coefficients: $\Gamma_{C T}-\Gamma_{T C}, \Gamma_{T T}-\Gamma_{C C}$ and $\Gamma_{A T}-\Gamma_{G C}$. Inserting the theoretical expression (20) of $\Gamma_{i j}$, one gets for each of the three differences:

$$
\begin{aligned}
& \Gamma_{C T}-\Gamma_{T C}=Z_{C T-T C} b+h_{C T}-h_{T C} \\
& \Gamma_{T T}-\Gamma_{C C}=Z_{T T-C C} b+h_{T T}-h_{C C} \\
& \Gamma_{A T}-\Gamma_{G C}=Z_{A T-G C} b+h_{A T}-h_{G C}
\end{aligned}
$$

where the coefficients $Z$ are functions of the free energies $\Delta G^{0}$. Summing up the three above equations, one gets that the l.h.s. is vanishing, due to eq. (17) and the equality of the correlation coefficients for complementary dimers, which implies, using eq. (25), that the coefficients $Z$ are related:

$$
Z_{C T-T C}+Z_{T T-C C}+Z_{A T-G C}=0
$$

Let us emphasize that this relation is biological species independent, by virtue of eq. (25) valid for each biological species, and by the complementarity rule for $\Gamma_{i j}$.

Note also that relation (33) is automatically satisfied when plugging the theoretical expressions of the free energies of the dimers (i.e. in terms of the parameters $\alpha_{0}, \alpha_{1}$ and $\alpha_{2}$ ).

Analogously using eq. (26) and the complementarity rule we get

$$
Z_{C A-G T}+Z_{C T-G A}+Z_{C G-G C}=0
$$

Note that eq. (27) is satisfied identically from the parametrization (20) and the constraint (24).

We report in Table 3 and Table 4 the values of the coefficients $Z$ and their sum, calculated with the experimental free energies given by the different authors (see table 6). As it can be seen most of the values of the sums are quite close to zero, except for Breslauer, SantaLucia and Sugimoto. 
Table 3: Values of the coefficients $Z$ of eq. (333).

\begin{tabular}{|l|rcrrrrrr|}
\hline & Gotoh & Vologodskii & Breslauer & Delcourt & SantaLucia & Sugimoto & Unified & Benight \\
\hline$Z_{C T-T C}$ & -0.123 & -0.115 & 0.133 & 0.060 & -0.498 & 0.125 & 0.027 & 0.005 \\
$Z_{T T-C C}$ & 0.318 & 0.220 & 0.492 & 0.160 & 0.268 & 0.375 & 0.318 & 0.080 \\
$Z_{A T-G C}$ & -0.285 & -0.205 & 0.145 & -0.180 & -0.560 & 0 & -0.155 & 0.015 \\
sum & -0.090 & -0.100 & 0.770 & 0.040 & -0.790 & 0.500 & 0.190 & 0.100 \\
\hline
\end{tabular}

Table 4: Values of the coefficients $Z$ of eq. (34)

\begin{tabular}{|l|rrrrrrrr|}
\hline & \multicolumn{1}{|c}{ Gotoh } & Vologodskii & Breslauer & Delcourt & SantaLucia & Sugimoto & Unified & Benight \\
\hline$Z_{C A-A C}$ & -0.013 & 0.025 & 1.013 & -0.110 & 0.358 & 0.425 & -0.077 & 0.405 \\
$Z_{C T-T C}$ & -0.123 & -0.115 & 0.133 & 0.060 & -0.498 & 0.125 & 0.027 & 0.005 \\
$Z_{C G-G C}$ & 0.035 & 0.010 & 0.995 & 0.010 & -0.300 & 0.850 & -0.110 & 0.150 \\
sum & -0.100 & -0.080 & 2.140 & -0.040 & -0.440 & 1.400 & -0.160 & 0.560 \\
\hline
\end{tabular}

\section{Conclusions}

We have proposed a 3 parameter formula to fit the free energy for the DNA dinucleotides and derived a set of sum rules. We have compared the theoretical values with the experimental data of seven authors as well as their averaged value. The results of the fits reported in Tables 7 and 2 show in the average a satisfactory agreement, except for Breslauer. On the basis of the above comparison, we cannot make any statement on the reliability of the different experimental data. In order to support our analysis by general theoretical arguments, we postulate a relation between the free energy and the dimer correlation function eq.(20), which has theoretical motivation from statistical mechanics as well as experimental motivation from the analysis of the computed correlation function. Our postulated equation satisfies the identity that the sum of correlation functions has to satisfy by definition. From consistency equation, we derive a set of sum rules for the correlation functions which are well satisfied by the computed values for several biological species. This analysis supports the validity of our relation eq. (20), which allows us to perform biological independent consistency checks, which is remarkably verified by our theoretical formula. We have checked which set of experimental data satisfy the consistency relations. The result is that the data of [8], [10] and [11] are not consistent. Therefore we disagree with the conclusions of [2]. The results of our analysis are more close to the ones of [1].

\section{References}

[1] J. SantaLucia, Proc. Natl. Acad. Sci. USA 95 (1998) 1460-1465.

[2] P. Miramontes and G. Cocho, Physica A 321 (2003) 577-586.

[3] L. Frappat, A. Sciarrino, P. Sorba, Phys. Lett. A 250 (1998) 214-221.

[4] L. Frappat, A. Sciarrino, P. Sorba, J. Biol. Phys. 27 (2001) 1-34.

[5] M.J. Serra and D.H. Turner, Methods Enzymol. 259 (1995) 242-261.

[6] O. Gotoh and Y. Tagashira, Biopolymers 20 (1981) 1033-1042. 
[7] A.V. Vologodskii, B.R. Amirikyan, Y.L. Lyubchenko and M.D. Frank-Kamenetskii, J. Biomol. Struct. Dyn. 2 (1984) 131-148.

[8] K.J. Breslauer, R. Frank, H. Blocker and L.A. Marky, Proc. Natl. Acad. Sci. USA 83 (1986) 9373-9377.

[9] S.G. Delcourt and R.D. Blake, J. Biol. Chem. 266 (1991) 15160-15169.

[10] J. SantaLucia, H. Allawi and P.A. Seneviratne, Biochemistry 35 (1996) 3555-3562.

[11] N. Sugimoto, S. Nakano, S. Yonemaya and K. Honda, Nucleic Acids Res. 24 (1996) 4501-4505.

[12] H.T. Allawi and J. SantaLucia, Biochemistry 36 (1997) 10581-10594.

[13] R. Owczarzy, P.M. Vallone, R.F Goldstein and A.S. Benight, Biopolymers 52 (1999) 29-56.

[14] M.J. Doktycz, R.F. Goldstein, T.M. Paner, F.J. Gallo and A.S. Benight, Biopolymers 32 (1992) 849864. 
Table 5: Dimer representation content.

\begin{tabular}{|ccccc||ccccc|}
\hline dimer & $J_{H}$ & $J_{V}$ & $J_{3 H}$ & $J_{3 V}$ & dimer & $J_{H}$ & $J_{V}$ & $J_{3 H}$ & $J_{3 V}$ \\
\hline CC & 1 & 1 & 1 & 1 & GC & 1 & 1 & 1 & 0 \\
CT & 0 & 1 & 0 & 1 & GT & 0 & 1 & 0 & 0 \\
CG & 1 & 0 & 1 & 0 & GG & 1 & 1 & 1 & -1 \\
CA & 0 & 0 & 0 & 0 & GA & 0 & 1 & 0 & -1 \\
TC & 1 & 1 & 0 & 1 & AC & 1 & 1 & 0 & 0 \\
TT & 1 & 1 & -1 & 1 & AT & 1 & 1 & -1 & 0 \\
TG & 1 & 0 & 0 & 0 & AG & 1 & 1 & 0 & -1 \\
TA & 1 & 0 & -1 & 0 & AA & 1 & 1 & -1 & -1 \\
\hline
\end{tabular}

Table 6: Experimental absolute values for dimer free energy parameters $\Delta G^{0}$ (in $\mathrm{kcal} / \mathrm{mol}$ ).

\begin{tabular}{|l|cccccccc|}
\hline & $\begin{array}{c}\text { Gotoh } \\
{[\mathbf{6}}\end{array}$ & $\begin{array}{c}\text { Vologodskii } \\
{[\mathbf{7}]}\end{array}$ & $\begin{array}{c}\text { Breslauer } \\
{[\mathbf{8}]}\end{array}$ & $\begin{array}{c}\text { Delcourt } \\
{[\mathbf{9}]}\end{array}$ & $\begin{array}{c}\text { SantaLucia } \\
{[10]}\end{array}$ & $\begin{array}{c}\text { Sugimoto } \\
{[1]}\end{array}$ & $\begin{array}{c}\text { Unified } \\
{[12]}\end{array}$ & $\begin{array}{c}\text { Benight } \\
{[13]}\end{array}$ \\
\hline $\mathrm{AA} / \mathrm{TT}$ & 0.43 & 0.89 & 1.66 & 0.67 & 1.02 & 1.20 & 1.00 & 0.91 \\
$\mathrm{AT} / \mathrm{TA}$ & 0.27 & 0.81 & 1.19 & 0.62 & 0.90 & 0.90 & 0.88 & 0.83 \\
$\mathrm{TA} / \mathrm{AT}$ & 0.22 & 0.76 & 0.76 & 0.70 & 0.90 & 0.90 & 0.58 & 0.68 \\
$\mathrm{CA} / \mathrm{GT}$ & 0.97 & 1.37 & 1.80 & 1.19 & 1.70 & 1.70 & 1.45 & 1.54 \\
$\mathrm{GT} / \mathrm{CA}$ & 0.98 & 1.35 & 1.13 & 1.28 & 1.43 & 1.50 & 1.44 & 1.25 \\
$\mathrm{CT} / \mathrm{GA}$ & 0.83 & 1.16 & 1.35 & 1.17 & 1.16 & 1.50 & 1.28 & 1.28 \\
$\mathrm{GA} / \mathrm{CT}$ & 0.93 & 1.25 & 1.41 & 1.12 & 1.46 & 1.50 & 1.30 & 1.30 \\
$\mathrm{CG} / \mathrm{GC}$ & 1.70 & 1.99 & 3.28 & 1.87 & 2.09 & 2.80 & 2.17 & 1.87 \\
$\mathrm{GC} / \mathrm{CG}$ & 1.64 & 1.96 & 2.82 & 1.85 & 2.28 & 2.30 & 2.24 & 1.86 \\
$\mathrm{GG} / \mathrm{CC}$ & 1.22 & 1.64 & 2.75 & 1.55 & 1.77 & 2.10 & 1.84 & 1.85 \\
\hline
\end{tabular}

Table 7: Fitted absolute values for dimer free energy parameters $\Delta G^{0}$ (in kcal $/ \mathrm{mol}$ ).

\begin{tabular}{|l|cccccccc|}
\hline & Gotoh & Vologodskii & Breslauer & Delcourt & SantaLucia & Sugimoto & Unified & Benight \\
\hline AA/TT & 0.46 & 0.92 & 1.13 & 0.75 & 1.08 & 1.11 & 0.93 & 0.85 \\
AT/TA & 0.30 & 0.79 & 0.93 & 0.65 & 0.91 & 0.93 & 0.78 & 0.81 \\
TA/AT & 0.30 & 0.79 & 0.93 & 0.65 & 0.91 & 0.93 & 0.78 & 0.81 \\
CA/GT & 1.02 & 1.40 & 1.94 & 1.26 & 1.57 & 1.75 & 1.51 & 1.36 \\
GT/CA & 1.02 & 1.40 & 1.94 & 1.26 & 1.57 & 1.75 & 1.51 & 1.36 \\
CT/GA & 0.85 & 1.27 & 1.73 & 1.16 & 1.40 & 1.57 & 1.35 & 1.33 \\
GA/CT & 0.85 & 1.27 & 1.73 & 1.16 & 1.40 & 1.57 & 1.35 & 1.33 \\
CG/GC & 1.73 & 2.01 & 2.94 & 1.88 & 2.24 & 2.57 & 2.25 & 1.90 \\
GC/CG & 1.73 & 2.01 & 2.94 & 1.88 & 2.24 & 2.57 & 2.25 & 1.90 \\
GG/CC & 1.25 & 1.61 & 2.34 & 1.57 & 1.73 & 2.03 & 1.78 & 1.81 \\
\hline
\end{tabular}

